\title{
The neutral $\rho$ and $A$ mesons in a strong magnetic field in SU(2) lattice gauge theory.
}

\section{E.V. Luschevskaya*}

Institute of Theoretical and Experimental Physics, B. Cheremushkinskaya, 25, Moscow, 117218, Russia

E-mail: luschevskaya@itep.ru

\section{O.V. Larina}

Institute of Theoretical and Experimental Physics, B. Cheremushkinskaya, 25, Moscow, 117218, Russia

E-mail: olarina@itep.ru

\begin{abstract}
The correlators of vector, axial and pseudoscalar currents have been calculated in the external strong magnetic field in SU(2) gluodynamics on the lattice. The masses of the neutral $\rho$ and $A$ mesons with different spin projections $s=0, \pm 1$ to the axis parallel to the external magnetic field $B$ have been calculated. The masses of the neutral axial and vector mesons with zero spin $s=0$ decrease with the growth of the magnetic field and the masses with $s= \pm 1$ increase with the magnetic field. The mass extrapolations were performed.
\end{abstract}

31st International Symposium on Lattice Field Theory - LATTICE 2013

July 29 - August 3, 2013

Mainz, Germany

${ }^{*}$ Speaker. 


\section{Introduction}

Magnetic fields of the order of $\sim 2 \mathrm{GeV}$ existed in the early Universe during the electroweak transition [1]. The values of the magnetic fields in the non-central heavy-ion collisions can reach the value $15 m_{\pi}^{2} \sim 0.29 \mathrm{GeV}^{2}$ [2].

STAR collaboration has detected the chiral magnetic effect at RHIC in the non-central collisions of gold ions $[3,4,5]$. Later this effect was also observed in the experiment ALICE at LHC [6]. The strong magnetic field also results to the modification of the phase diagram of QCD. Phenomenological models show that the critical temperature of the transition between the phases of confinement and deconfinement varies with increasing of the external magnetic field $B$, and the phase transition becomes of first order [7].

The growth of the phase transition temperature $T_{c}$ was predicted by the models of Nambu-JonaLasinio type: NJL, EPNJL, PNJL [8] and PNJL $_{8}$ [9], the Gross-Neveu model [10, 11], as well as the first calculations on the lattice QCD with two quarks [12]. However, the lattice calculations in QCD with $N_{f}=2+1$ revealed that $T_{c}$ decreases with increasing of $B$ value [13]. The chiral perturbation theory gives the decrease of $T_{c}$ with the growth of field value [14].

It has been shown in the framework of the Nambu-Jona-Lasinio model that in the presence of sufficiently strong magnetic fields $\left(B_{c}=m_{\rho}^{2} / e \simeq 10^{16} \mathrm{~T}\right) \mathrm{QCD}$ vacuum becomes a superconductor [15] along the direction of the magnetic field. The transition to the superconducting phase is accompanied by a condensation of the charged $\rho$ mesons. Calculations on the lattice [16] also indicate the existence of the superconducting phase. We have investigated the behavior of the masses of the neutral $\rho$ with different spin projection $s=0$ and $s= \pm 1$ to the direction of the magnetic field. Quark propagators were calculated with the chiral invariant fermionic operator. In [17] the mass of neutral vector $\rho$ meson was calculated in the relativistic quark-antiquark model, the mass of neutral $\rho$ meson with zero spin does not vanishes with the growth of the magnetic field in the confinement phase in a contradiction with the results of [15].

\section{Details of the calculations}

The improved Symanzik action has been used for the generation of $S U(2)$ gauge field configurations similarly to our previous work [18]. The calculations were performed on symmetric lattices with different lattice volumes $14^{4}, 16^{4}, 18^{4}$ and lattice spacings $a=0.0681,0.0998$ and 0.138348 fm.

Fermionic spectrum in the background of $S U(2)$ gauge fields were calculated using a chiral invariant overlap operator, proposed by Neuberger [19]. This operator allows to explore the theory without chiral symmetry breaking.

In a continuous space the analogue of this operator is the Dirac operator $D=\gamma^{\mu}\left(\partial_{\mu}-i A_{\mu}\right)$, the corresponding Dirac equation is

$$
D \psi_{k}=i \lambda_{k} \psi_{k} .
$$

The Neuberger overlap operator allows to calculate the eigenfunctions $\psi_{k}$ and the eigenvalues $\lambda_{k}$ for a test quark in an external gauge field configurations $A_{\mu} . A_{\mu}$ is a sum of $S U(2)$ gauge fields and the external abelian uniform magnetic field. From the eigenfunctions of the Dirac operator we construct operators and correlators. 
Abelian fields interact with quarks, so for the introduction of the external magnetic field it's necessary to perform the following substitution

$$
\begin{gathered}
A_{\mu i j} \rightarrow A_{\mu i j}+A_{\mu}^{B} \delta_{i j}, \\
A_{\mu}^{B}(x)=\frac{B}{2}\left(x_{1} \delta_{\mu, 2}-x_{2} \delta_{\mu, 1}\right) .
\end{gathered}
$$

To match this change with the lattice boundary conditions the twisted boundary conditions for fermions have been used as described in [20].

The value of magnetic field on the lattice is quantized

$$
q B=\frac{2 \pi k}{(a L)^{2}}, \quad k \in \mathbb{Z},
$$

where $q=-1 / 3 e$ is the charge of $d$-quark, there is one type of fermions in the theory, $a$ is the lattice spacing in physical units. The quantization condition imposes the limit on the minimum value of the magnetic field. For our calculations it equals to $0.386 \mathrm{GeV}^{2}$ for lattice volume $16^{4}$ and lattice spacing $0.1383 \mathrm{fm}$.

For each value of the quark mass in the interval $m_{q} a=0.01-0.8$ statistical independent configurations of the gluon field have been used.

\section{Calculation of the observables}

The following observables were calculated

$$
\left\langle\psi^{\dagger}(x) O_{1} \psi(x) \psi^{\dagger}(y) O_{2} \psi(y)\right\rangle_{A}
$$

where $O_{1}, O_{2}=\gamma_{5}, \gamma_{\mu, v}$ are Dirac gamma matrices, $\mu, v=1, . .4$. In the Euclidean space $\psi^{\dagger}=\bar{\psi}$ [21]. The correlators (3.1) are defined by the Dirac propagators, for their calculation the inverse matrix for the massive Dirac operator $1 /(D+m)$ should be found. For M lowest eigenstates Dirac operator it is represented by the sum

$$
\frac{1}{D+m}(x, y)=\sum_{k<M} \frac{\psi_{k}(x) \psi_{k}^{\dagger}(y)}{i \lambda_{k}+m} .
$$

In this work $M=50$ was used. On the lattice in the background of a gauge field $A_{\mu}$ the observables (3.1) have the form

$$
\left\langle\bar{\psi} O_{1} \psi \bar{\psi} O_{2} \psi\right\rangle_{A}=\sum_{k, p<M} \frac{\left\langle k\left|O_{1}\right| k\right\rangle\left\langle p\left|O_{2}\right| p\right\rangle-\left\langle p\left|O_{1}\right| k\right\rangle\left\langle k\left|O_{2}\right| p\right\rangle}{\left(i \lambda_{k}+m\right)\left(i \lambda_{p}+m\right)}
$$

The first term in the numerator represents a disconnected part, and the second one with a minus sign - a connected part. The first term is less than the second one, has large statistical errors, does not affect the result, so for further calculations only the connected part of the correlator was used.

The mass of the neutral $\rho$ meson was obtained from the correlator of vector currents $\left\langle j_{\mu}^{V}(x) j_{v}^{V}(y)\right\rangle_{A}$, where $j_{\mu}^{V}(x)=\psi^{\dagger}(x) \gamma_{\mu} \psi(x)$. The correlator $\left\langle j^{P S}(x) j^{P S}(y)\right\rangle_{A}$ gives the mass of the $\pi$ meson, where $j^{P S}=\psi^{\dagger}(x) \gamma_{5} \psi(x)$ is the pseudoscalar current. 
For the calculation of meson masses we used the method, based on the spectral expansion of the lattice correlation function

$$
\begin{gathered}
C\left(n_{t}\right)=\left\langle\psi^{\dagger}\left(\overrightarrow{0}, n_{t}\right) O_{1} \psi\left(\overrightarrow{0}, n_{t}\right) \psi^{\dagger}(\overrightarrow{0}, 0) O_{2} \psi(\overrightarrow{0}, 0)\right\rangle_{A}=\sum_{k}\left\langle 0\left|O_{1}\right| k\right\rangle\left\langle k\left|O_{2}^{\dagger}\right| 0\right\rangle e^{-n_{t} a E_{k}}, \\
C\left(n_{t}\right)=A_{0} e^{-n_{t} a E_{0}}+A_{1} e^{-n_{t} a E_{1}}+\ldots,
\end{gathered}
$$

where $A_{0}, A_{1}$ are some constants, $E_{0}$ is the energy of the lowest state, for the particle with average zero momentum $\vec{p}=0$ its energy coincides with its mass $E_{0}=m_{0}, E_{1}$ is the energy of the first excited state, $a$ is the lattice spacing, $n_{t}$ is the time coordinate on the lattice. From the expansion (3.5) one can see that for large values $n_{t}$ the main contribution comes from the ground energy state.

Due to the periodic boundary conditions the contribution of the ground state into the propagator of a meson has the form

$$
f\left(n_{t}\right)=A_{0} e^{-n_{t} a E_{0}}+A_{0} e^{-\left(N_{T}-n_{t}\right) a E_{0}}=2 A_{0} e^{-N_{T} E_{0} / 2} \cosh \left(\left(N_{T}-n_{t}\right) a E_{0}\right)
$$

The mass value of the ground state mass can be obtained, fitting the correlator by the function (3.6), $n_{t}$ is the number at lattice site in the time direction.

The second method which we use is the Maximal Entropy Method (MEM) [22]. The imaginarytime Euclidean correlator $G(\tau, \vec{p})=\int d^{3} x\left\langle O(\tau, \vec{x}) O^{\dagger}(0, \overrightarrow{0})\right\rangle e^{-i \vec{p} \vec{x}}$ is related to the spectral function $\rho(\omega, \vec{p})$ according to

$$
G(\tau, \vec{p})=\int_{0}^{\infty} \frac{d \omega}{2 \pi} K(\tau, \omega) \rho(\omega, \vec{p})
$$

In general $\rho(\omega, \vec{p})$ contains the all properties of mesons and hadrons. We consider zero momentum case $\langle\vec{p}\rangle=0$ and drop the momentum dependence in the following. The first peak in the spectral function corresponds to the energy of the ground state. The kernel in (3.7) is given by

$$
K(\tau, \omega)=\frac{\cosh [\omega(\tau-1 / 2 T)]}{\sinh (\omega / 2 T)}
$$

where $T$ is the temperature, $\tau$ is the euclidean time, $\omega$ is the frequency. For the calculation of the spectral function an inversion of (3.7) has to be performed.

On the lattice this problem is ill-defined, because the correlator $G(\tau)$ is calculated numerically at the discrete set of points $\tau_{i}=\tau_{\min }+(i-1) a, i=1, \ldots N_{\tau}$ and $N_{\tau}=1 /(a T)$ is typically of the order of $O(10)$. The integral was approximated by a discrete sum at the points $\omega_{n}=n \triangle \omega, n=1, \ldots, N_{\omega}$ and $N_{\omega}$ is usually $\sim O\left(10^{3}\right)$. We cut off the integral (3.7) at some $\omega_{\max }$. All the same the inversion becomes impossible. But the ideas of Bayesian probability theory allow to overcome this difficulty.

The most probable spectral function $\rho(\omega)$ can be constructed if we find the maximum of the conditional probability $P[\rho \mid D H \alpha m]$, where $D$ is the data, $H$ is our hypothesis, $\alpha$ is a real and positive parameter, $m=m(\omega)$ is a default model. This procedure is equivalent to a maximization of the free energy $F=L-\alpha S$, where $S$ is the Shannon entropy term, defined by the following way

$$
S=\int_{0}^{\infty} d \omega\left[\rho(\omega)-m(\omega)-\rho(\omega) \ln \frac{\rho(\omega)}{m(\omega)}\right] .
$$

$L$ is the standard likelihood function, the detailed explanation how to make a corresponding discretization on the lattice is given in [22]. The parameter $\alpha$ balances the relative importance of the 
data and the prior hypothesis. We take $\alpha \in\left[\alpha_{\min }, \alpha_{\max }\right]$ and average the data over this interval. This interval was choosen in such way that the results vary slightly, for approximately $10 \%$.

The kernel (3.8) contains divergence at $\omega=0$ leading to the unstable behaviour of the procedure at small energies. The Bryan's key idea was to redefine the kernel and spectral function

$$
\bar{K}(\omega, \tau)=\frac{\omega}{2 T} K(\omega, \tau), \quad \bar{\rho}(\omega)=\frac{2 T}{\omega} \rho(\omega),
$$

so that $K(\omega, \tau) \rho(\omega)=\bar{K}(\omega, \tau) \bar{\rho}(\omega)$ and apply the SVD theorem to the modified discretized kernel $\bar{K}\left(\omega_{n}, \tau_{i}\right)$, see [23]. We use this modified algorithm to determine the spectral function in the form

$$
\bar{\rho}(\omega)=\bar{m}(\omega) \exp \sum_{i=1}^{N} \bar{c}_{i} \bar{u}_{i}(\omega)
$$

The column vectors $u_{i},(i=1, . ., N)$ are normalized

$$
\left\langle u_{i} \mid u_{j}\right\rangle \equiv \sum_{n=1}^{N_{\omega}} u_{i}\left(\omega_{n}\right) u_{j}\left(\omega_{n}\right)=\delta_{i j}
$$

$c_{i}$ are the coefficients and we set $\bar{K}(0, \tau)=1$.

To reconstruct the spectral function $\rho(\omega)$ we have to choose the default model $\bar{m}(\omega)$ in a correct way. The default model should describe correctly the high and low energy behaviour of the spectral function. Following the analysis of [24] we choose it in the form

$$
\bar{m}(\omega)=m_{a} \omega+m_{b}, \quad m_{a}=\frac{G\left(N_{\tau} / 2\right)}{T^{2}}, m_{b}=a_{H} \frac{3}{8 \pi^{2}},
$$

$a_{H}=1$ for scalar and pseudoscalar channel, $a_{H}=2$ for the vector and axial vector channel [25]. We also try another default model (constant function, $\sim \omega^{2}$, vary the $m_{a}$ and $m_{b}$ ), but the choice (3.13) gives the best convergence for MEM.

\section{Results}

At first we calculate the mass of the neutral $\pi$ meson on the lattice from the correlators of the pseudoscalar currents $C^{P S P S}\left(n_{t}\right)=\left\langle j^{P S}\left(\overrightarrow{0}, n_{t}\right) j^{P S}(\overrightarrow{0}, 0)\right\rangle_{A}$, where $j^{P S}\left(\overrightarrow{0}, n_{t}\right)=\bar{\psi}\left(\overrightarrow{0}, n_{t}\right) \gamma_{5} \psi\left(\overrightarrow{0}, n_{t}\right)$. At Fig.1 (left) the squared pion mass is depicted for $16^{4}$ lattice volume, different bare quark masses and different values of the magnetic field $H=\sqrt{e B}$. Fig.1 (left) reveals the linear dependence of the squared $\pi$ meson mass versus the bare quark mass. The Chiral Perturbation Theory (ChPT) predicts the linear dependence according to the relation

$$
f_{\pi}^{2} m_{\pi}^{2}=m_{q}^{r e n}\langle\bar{\psi} \psi\rangle
$$

where $f_{\pi}$ is the pion decay constant, $\langle\bar{\psi} \psi\rangle$ is the chiral condensate, $m_{q}^{r e n}$ is the renormalized quark mass. In the limit of zero quark mass the pions are massless. The pion masses are slightly shifted relatively zero due to the quark mass renormalization on the lattice. The value of the shift corresponds to the quark mass renormalization.

Fig.1 (right) represents the $\pi$ meson mass versus the value of the squared magnetic field after the quark mass renormalization. For the renormalized pion mass we get the linear mass dependence 

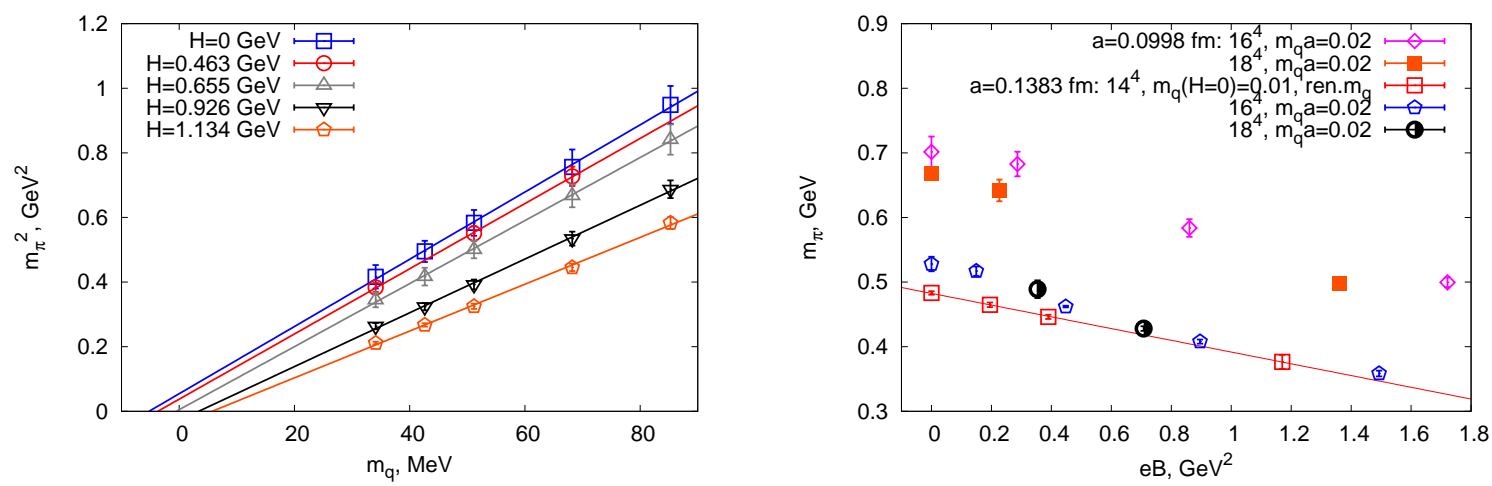

Figure 1: The squared mass of the neutral $\pi$ meson extracted from the pseudoscalar correlator $C^{P S P S}\left(n_{t}\right)$ versus the bare lattice quark mass for the lattice volume $16^{4}$, lattice spacing $0.1155 \mathrm{fm}, \beta=3.2000$ and different values of the external magnetic field (left). The mass of the neutral $\pi$ meson depending on the squared value of magnetic field for renormalized and nonrenormalized quark mass (right). The all results were obtained by coshinus function fit.

from the magnetic field, the slope is negative in accordance with the results of A.Smilga, obtained with the Chiral Perturbation Theory [26]. The value of the slope is smaller and differs from ChPT, because we explore the $S U(2)$ gauge theory without dinamical quarks.

At Fig.2 (left) the $\rho$ meson mass versus the bare quark mass is represented at zero external magnetic field. The extrapolation to the infinite physical volume have been performed. The masses are calculated from the correlators of vector currents, the symmetry between the different spatial directions has been taken into account, thereby we improve statistics in three times. The quark mass renormalization have not considered because it is very small at zero magnetic field. We extrapolate $m_{\rho}$ to the quark mass $m_{q_{0}}$ corresponding to the physical value of the $\pi$ meson mass equal $135 \mathrm{MeV}$. The vector meson mass $m_{\rho} \simeq 980 \pm 30 \mathrm{MeV}$ for the lattice spacing $a=0.1338 \mathrm{fm}$ and $1020 \pm 20$ $\mathrm{MeV}$ for $a=0.1155 \mathrm{fm}$ in $S U(2)$ gluodynamics.

If the external magnetic field is directed along the third coordinate axis then the meson masses with zero spin projection to the direction of the external magnetic field are calculated from the expression (3.3) with $O_{1}, O_{2}=\gamma_{3}$. Fig.2 (right) shows the mass of the neutral vector meson with zero spin obtained by the Maximal Entropy Method at different lattice volumes, spacings and bare quark masses. The mass decreases with the growth of the magnetic field for the all sets of data. Errors were calculated taking into account the $\omega$-discretization.

The masses of the vector meson were calculated for various values of the magnetic field. For the nonzero magnetic field the quark mass renormalization $\delta m_{\text {latt }}^{\text {ren }}$ has been taken into account. It depends on the lattice volume, lattice spacing and the magnetic field value. Therefore we calculate the $\pi$ meson mass for various values of $m_{q}$ (the bare quark mass which enter into lattice lagrangian). We extrapolate the mass to small values of $m_{q}$, fix the value of the bare quark mass $m_{q_{0}}$ corresponding to the physical value of the $\pi$ meson mass at zero magnetic field (135 Mev). We calculate the $\rho$ mass for several values of $m_{q}$ in the interval $m_{q}=0.01 \div 0.8$, perform fits and find the coefficients $a_{i}$ and $b_{i}$ in the equations

$$
m_{\rho}(s=0)=a_{0}+a_{1} m_{q}
$$



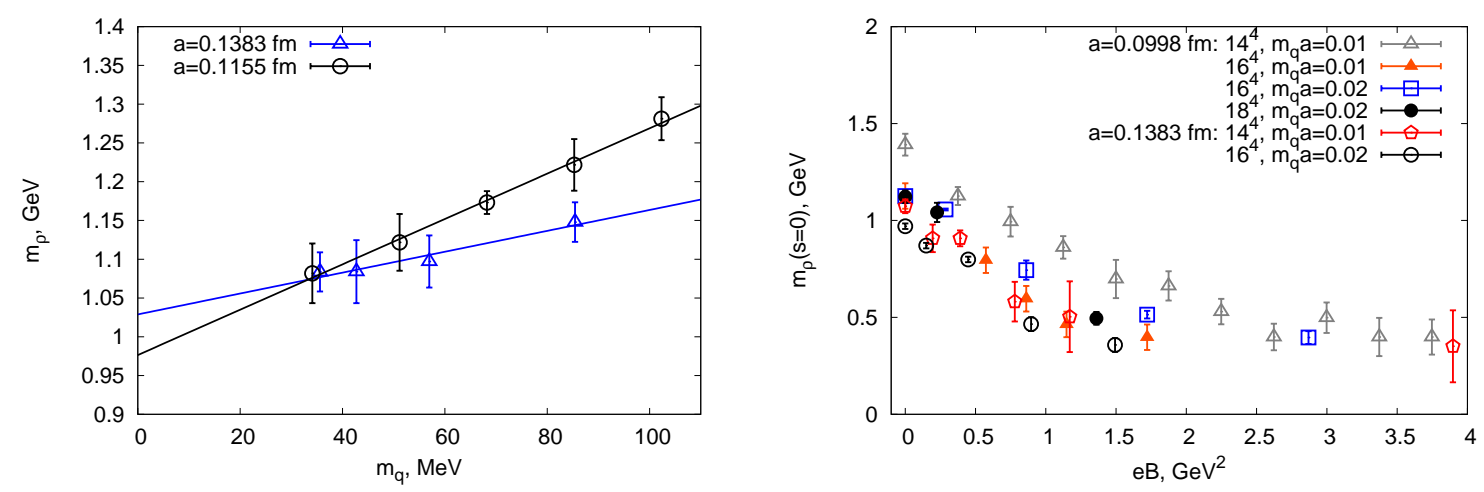

Figure 2: The mass of the neutral $\rho$ meson for the different quark masses and two lattice spacings obtained by coshinus function fit. The extrapolation was performed to the physical pion meson mass $m_{\pi}=135 \mathrm{MeV}$ (left). The mass of the neutral vector $\rho$ meson with zero spin $s=0$ depending on the magnetic field value for the lattice volumes $14^{4}, 16^{4}, 18^{4}$ and lattice spacings $a=0.0998,0.1155 \mathrm{fm}$, obtained by the Maximal Entropy Method (right).
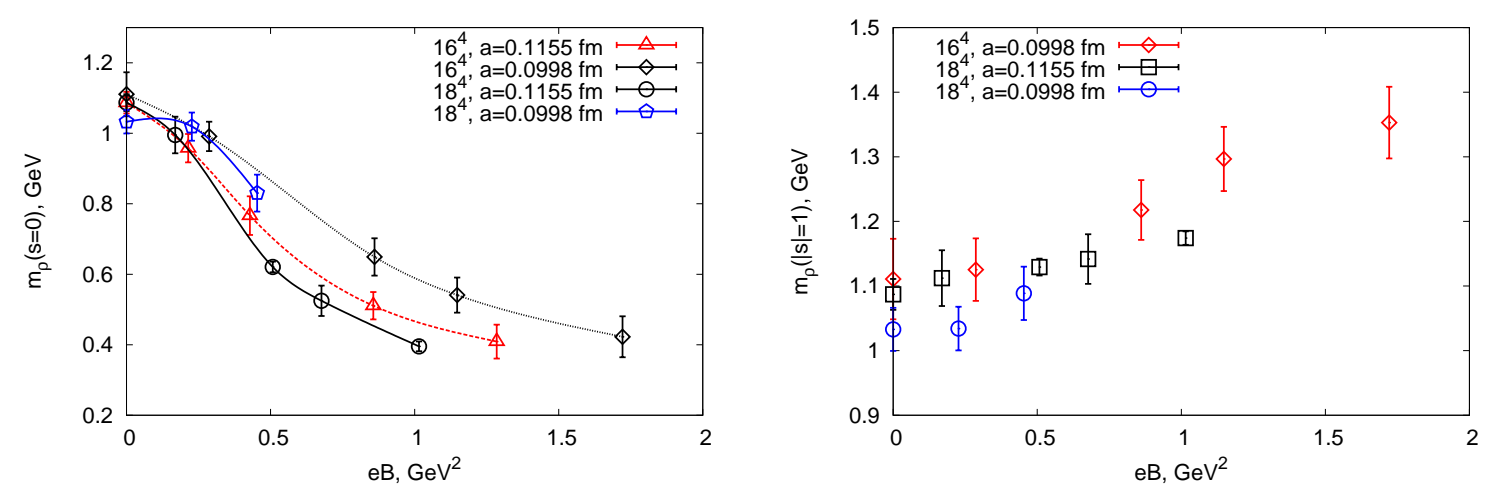

Figure 3: The mass of the neutral vector $\rho$ meson with spin $s=0$ depending on the external magnetic field for the lattice volumes $16^{4}, 18^{4}$ and lattice spacings $a=0.0998,0.1155 \mathrm{fm}$ (left). The mass of the neutral vector $\rho$ meson with spin $s= \pm 1$ versus the magnetic field value for the lattice volumes $16^{4}, 18^{4}$ and lattice spacings $a=0.0998,0.1155 \mathrm{fm}$ (right). The results were obtained after quark mass extrapolation by coshinus function fit.

$$
m_{A}(s=0)=b_{0}+b_{1} m_{q}
$$

and then extrapolate $m_{\rho}\left(m_{q}\right)$ to the physical values $m_{\rho}\left(m_{q_{0}}\right)$ at $m_{q}=m_{q_{0}}$ using (4.2) and (4.3).

Different components of the correlators of vector currents were calculated, diagonal components are essentially nonzero, while nondiagonal ones are zero within the error bars. The correlators of vector currents perpendicular to the magnetic field are $C_{11}^{V V}\left(n_{t}\right)=\left\langle j_{1}^{V}\left(\overrightarrow{0}, n_{t}\right) j_{1}^{V}(\overrightarrow{0}, 0)\right\rangle_{A}$ and $C_{22}^{V V}\left(n_{t}\right)=\left\langle j_{2}^{V}\left(\overrightarrow{0}, n_{t}\right) j_{2}^{V}(\overrightarrow{0}, 0)\right\rangle_{A}$, where $j_{1}^{V}\left(\overrightarrow{0}, n_{t}\right)=\bar{\psi}(\overrightarrow{0}, 0) \gamma_{1} \psi\left(\overrightarrow{0}, n_{t}\right)$ and $j_{2}^{V}\left(\overrightarrow{0}, n_{t}\right)=\bar{\psi}(\overrightarrow{0}, 0) \gamma_{2} \psi\left(\overrightarrow{0}, n_{t}\right)$. The masses with spin $s= \pm 1$ are found from the relations $C^{V V}(s=1)=\left(C_{11}^{V V}+i C_{22}^{V V}\right) / \sqrt{2}$ and $C^{V V}(s=-1)=-\left(C_{11}^{V V}-i C_{22}^{V V}\right) / \sqrt{2}$.

At Fig.3 (left) the mass of the neutral $\rho$ meson with zero spin is shown depending on the magnetic field value. For the all lattice volumes $16^{4}, 18^{4}$ and spacings $a=0.0998,0.11558 \mathrm{fm}$ 

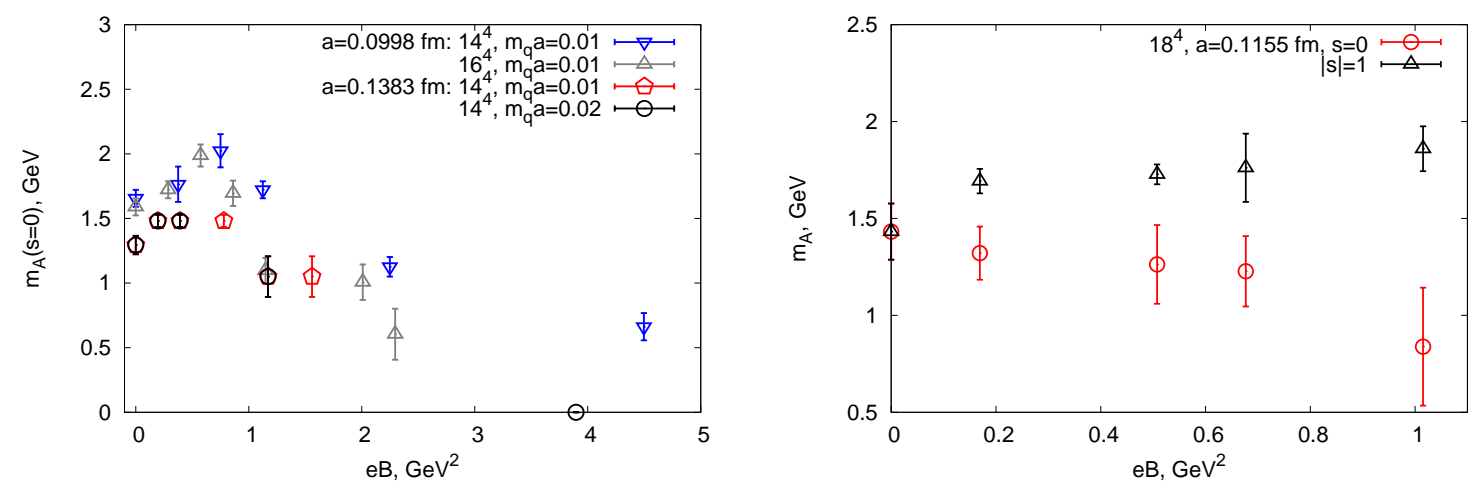

Figure 4: The mass of the neutral axial $A$ meson with zero spin $s=0$ versus the value of the external magnetic field for the lattice volumes $14^{4}$ and $16^{4}$ and different lattice spacings, obtained by the Maximal Entropy Method (left). The mass of the neutral axial $A$ meson with different spins depending on the magnetic field value for the lattice volumes $16^{4}, 18^{4}$ and lattice spacings $a=0.0998,0.1155 \mathrm{fm}$. The data were obtained by coshinus function fit (right).

the mass decreases with the magnetic field. The points are connected by splines to guide the eyes. Fig.3 (right) shows the mass of the $\rho$ meson mass with nonzero spin versus the field value. The masses with spin $s= \pm 1$ increase with the field. The results were obtained after the quark mass extrapolation.

We observe a weak dependence of masses from the lattice volume and lattice spacings, but the qualitative behaviour of the masses with the magnetic field is the same.

Fig. 4 (left) shows the behaviour of the neutral axial meson mass with zero spin depending on the external magnetic field calculated by the Maximal Entropy Method.

At Fig. 4 (right) we see the mass of the neutral axial meson with zero spin $s=0$ and nonzero spin projections $s= \pm 1$ to the direction of the magnetic field. The calculation of the axial meson mass needs much more statistics, than for the vector meson, especially for nonzero spin components. The mass of $A$ with zero spin decreases, the masses with $s= \pm 1$ increase slowly.

Unfortunately on the lattice in the presence of the magnetic field the quantum numbers of mesons are not precise. The mixing takes place due to the interaction between photons and vector (axial) quark currents and can occur between neutral pion and neutral $\rho$ or $A$ meson with zero spin. No severe methods occurs to disentangle these two states in the magnetic field. However we have indications that the masses of vector and axial mesons with $s= \pm 1$ definitely increase in our $S U$ (2) theory. The investigations of the mass behaviour in QCD with dynamical quarks present the huge interest.

\section{Conclusions}

In this work we explore the masses of the neutral $\pi, \rho$ and $A$ mesons in the background of the strong magnetic field of the hadronic scale in the confinement phase. The masses with zero spin projection to the magnetic field differ from the masses with spin projection $s= \pm 1$. The masses with $s=0$ decrease with the magnetic field, the masses with $s= \pm 1$ increase with the field. 
We consider this phenomena to be the result of the anisotropy, which the strong magnetic field creates. We do not observe any condensation of the neutral mesons, so there are no evidences of superfluidity in the confinement phase. However the presence of superconducting phase at high values of the magnetic field $B$ [27] in QCD is a hot topic for discussions. The condensation of charged $\rho$ mesons would be an evidence of the existence of the superconductivity in QCD.

The authors are grateful to ITEP supercomputer center (the calculations were performed at supercomputers "Graphin" and "Stakan"), Moscow Supercomputer JSCC Center. Athors are grateful to M.I.Polikarpov, M.N.Chernodub for the usefull discussions and comments.

\section{References}

[1] D. Grasso and H.R. Rubinstein, Phys. Rept. 348, 163 (2001), arXiv:astro-ph/0009061.

[2] V. Skokov, A. Illarionov and V. Toneev, Int. J. Mod. Phys. A24, 5925 (2009), arXiv:0907.1396 [nucl-th].

[3] S.A. Voloshin, Phys. Rev. C70, 057901 (2004), arXiv:hep-ph/0406311.

[4] S.A. Voloshin and STAR Collaboration, Phys. Rev. D78, 074033 (2008), arXiv:0808.3382; H.J. Warringa, arXiv:0906.2803; D.E. Kharzeev, arXiv:0906.2808, arXiv:0908.0314.

[5] G. Wang, arXiv:1210.5498 [nucl-ex].

[6] Y. Hori and ALICE Collaboration, arXiv:1211.0890 [nucl-ex]; ALICE Collaboration, Phys. Rev. Lett. 110, 012301 (2013).

[7] E.S. Fraga and A.J. Mizher, Phys. Rev. D78, 025016 (2008), arXiv:0804.1452; Nucl. Phys. A820, 103C (2009), arXiv:0810.3693; A.J. Mizher, M.N. Chernodub, and E.S. Fraga, Phys. Rev. D82, 105016 (2010), arXiv:1004.2712.

[8] R. Gatto and M. Ruggieri, Phys. Rev. D83, 034016 (2011), arXiv:1012.1291; R. Gatto and M. Ruggieri, Phys. Rev. D82, 054027 (2010), arXiv:1007.0790;

[9] K. Kashiwa, Phys. Rev. D83, 117901 (2011), arXiv:1104.5167.

[10] S. Kanemura, H.-T. Sato, H. Tochimura, Nucl. Phys. B517, 567 (1998), arXiv:hep-ph/9707285.

[11] K.G. Klimenko, Theor. Math. Phys. 90, 1 (1992).

[12] Massimo D’Elia, Swagato Mukherjee, Francesco Sanfilippo, Phys. Rev. D82, 051501 (2010), arXiv:1005.5365v2 [hep-lat].

[13] G.S. Bali, F. Bruckman, G. Endrodi, Z. Fodor, S.D. Katz, S. Krieg, A. Schafer, K.K. Szabo, JHEP 02, 044 (2012), arXiv:1111.4956 [hep-lat].

[14] N.O. Agasian and S.M. Fedorov, Phys. Lett. B663, 445 (2008), arXiv:0803.3156 [hep-ph].

[15] M.N. Chernodub, Phys. Rev. Lett. 106, 142003 (2011), arXiv:1101.0117v2 [hep-ph].

[16] V.V. Braguta, P.V. Buividovich, M.N. Chernodub, M.I. Polikarpov, arXiv:1104.3767 [hep-lat].

[17] M.A. Andreichikov, B.O. Kerbikov, V.D. Orlovsky, Yu.A. Simonov, arXiv:1304.2533 [hep-ph].

[18] V.G. Bornyakov, E.V. Luschevskaya, S.M. Morozov, M.I. Polikarpov, E.-M. Ilgenfritz, M. Muller-Preussker, Phys. Rev. D79, 054505 (2009), arXiv:0807.1980 [hep-lat].

[19] H. Neuberger, Phys. Lett. B417, 141 (1998), arXiv:hep-lat/9707022. 
[20] M.H. Al-Hashimi and U.J. Wiese, Annals of Phys. 324, 343 (2009), arXiv:0807.0630 [quant-ph].

[21] A.I. Vainshtein, V.I. Zakharov, V.A. Novikov and M.A. Shifman, Sov. Phys. Usp. 25, 195 (1982); Usp. Fiz. Nauk 136, 553 (1982).

[22] M. Asakawa, T. Hatsuda and Y. Nakahara, Prog. Part. Nucl. Phys. 46, 459 (2001).

[23] R.K. Bryan, Eur. Biophys. J18, 165 (1990).

[24] Gert Aarts, Chris Allton, Justin Foley, Simon Hands, Seyong Kim, Phys. Rev. Lett. 99: 022002 (2007), arXiv:hep-lat/0703008v2.

[25] F. Karsch et al, Phys. Lett. B497, 249 (2001).

[26] I. A. Shushpanov and A. V. Smilga, Phys. Lett. B402, 351 (1997).

[27] M.N. Chernodub, Phys. Rev. D82, 085011 (2010), arXiv:1008.1055 [hep-ph]. 